\title{
RESSEÇÃO ESPACIAL EM FOTOGRAMETRIA COM QUATÉRNIOS
}

\author{
The photogrammetric spatial resection using quaternions
}

\author{
Amanda Maria da Silva ${ }^{1}$ \\ Daniel Carneiro da Silva ${ }^{1}$ \\ 1Universidade Federal de Pernambuco. Programa de Pós-Graduação em Ciências Geodésicas e \\ Tecnologias da Geoinformação. Recife, Pernambuco, Brasil. Email:amanda27mendy@gmail.com; \\ danielcs@ufpe.br.
}

\section{Resumo:}

A fotogrametria usa comumente a equação da colinearidade com as rotações segundo os eixos cartesianos dadas com os ângulos de Euler. No entanto, podem ocorrer combinações desses ângulos que torna a matriz de rotação instável e as soluções podem não convergir ou serem indefinidas. Este problema, chamado de gimbal lock, é muito comum em robótica, visão por computadores e aeronáutica, quando é necessário definir a posição e orientação de uma câmara no espaço tridimensional e tem sido resolvido com a substituição dos ângulos de Euler pelo uso dos quatérnios. Este trabalho tem por objetivo usar esta solução para resolver os problemas de orientações críticas em fotogrametria nos casos de resseção espacial. Foram implementados programas com métodos iterativos e diretos com substituição dos ângulos de Euler pelos quatérnios para comparações com o métoda equação da colinearidade, usando dados de situações reais de medições obtidas com fotogrametria terrestre. Os diferentes testes e implementações efetuados mostraram as vantagens e desvantagens de cada um dos métodos e comprovou que os quatérnios são mais robustos, fornecem resultados mais confiáveis e permitem cálculos de resseção espacial de fotografias em posições com ambiguidades de rotações e situações críticas de gimbal lock.

Palavras-chave: Gimbal Lock, Quatérnios, Rotações, Fotogrametria.

\begin{abstract}
:
Photogrammetry basically uses the colinearity equation in which the rotations according to the Cartesian axis are given with the Euler angles. However, there may be angle combinations that leave the rotation matrix unstable and thus, the solution may not converge or even be undefined. This problem, called gimbal lock, is very common in robotics, computer vision and aeronautics, when its necessary to define the position and orientation of a chamber in tridimensional space and has been solved with the substitution of Euler angles by quaternions. This study aims to use this solution to solve critical photogrammetry orientation problems, in cases of spatial resection. Programs with iterative and direct methods with the substitution of Euler angles by quaternaries were implemented in order to compare against the colinearity method using data of a real situation of measurements obtained with terrestrial photogrammetry. The different implementations and tests made showed the advantages and disadvantages of both methods and
\end{abstract}


that the quaternions are more robust, get better results and allow spatial resection calculation of photographs in positions of rotation ambiguities and critical situations of gimbal lock.

Keywords: Gimbal Lock, Quaternions, Rotations, Photogrammetry.

\section{Introdução}

A resseção espacial em fotogrametria é um método de determinação dos parâmetros de orientação exterior de uma fotografia que são os ângulos de orientação $\omega, \varphi, \kappa$ e as coordenadas do centro perspectivo, $X_{r}, Y_{r}$ e $Z_{c}$, a partir de fotocoordenadas $\left(x_{i}, y_{i}\right)$, de pontos conhecidos do espaço objeto $\left(X_{i}, Y_{i}, Z_{i}\right)$, e da distância focal (f) (Lugnani, 1987). Existem vários métodos iterativos e diretos de determinação da resseção espacial. Nos iterativos usa-se o ajustamento pelo Método dos Mínimos Quadrados (MMQ) com o modelo funcional baseado na equação da colinearidade e ângulos de Euler. Porém com estes ângulos são possíveis várias combinações de rotações que resultam na mesma matriz, ou seja, numa mesma orientação dos eixos no espaço, originando assim situações ambíguas. O problema não ocorre nos casos comuns dos levantamentos aéreos com fotografias praticamente verticais, mas pode ocorrer em fotogrametria aérea inclinada e terrestre, sendo que estes casos são pouco discutidos na literatura e podem passar despercebidos dentro de um bloco de fototriangulação. No caso específico da resseção, as múltiplas possibilidades de resultados durante as operações de ajustamento pelo MMQ podem convergir para valores que não são corretos, não convergir ou apresentar confiabilidade baixa.

A indefinição de orientação no espaço é muito comum em Robótica, Visão computacional e Aeronáutica, sendo conhecida como gimbal lock, que é a perda de um grau de liberdade rotacional (Araújo, 2000). Do ponto de vista matemático isto equivale à questão em que, quando escolhida uma sequência de rotações, há uma situação de alinhamento de dois eixos em torno dos quais se realizam as rotações, fazendo com que nem todo o espaço das rotações possíveis possa ser atingido numa próxima sequência de rotações (Lavieri, 2011). A solução usual adotada nas áreas mencionadas é a substituição da representação dos ângulos de Euler pelos quatérnios, que são uma generalização dos números complexos para o campo tridimensional, com a vantagem de não acontecer o caso de instabilidade de rotações (Araújo, 2000 e Shih, 1990).

$\mathrm{O}$ assunto das instabilidades deve merecer mais atenção atualmente porque está havendo um grande avanço em aplicações de fotogrametria em sistemas terrestres móveis de mapeamento, em Veículos Aéreos Não Tripulados (VANTs) e sistemas aéreos com câmaras inclinadas, que criam situações fora dos padrões convencionais e podem gerar situações críticas quanto à estabilidade do ajustamento e do processamento.

Os objetivos deste artigo são identificar e avaliar as ocorrências de situações de orientações críticas da resseção espacial na fotogrametria, devido ao uso dos ângulos de Euler e analisar os resultados da substituição desses por quatérnios nas matrizes de rotação, a partir de resultados do trabalho mais completo de Silva (2014). Para isto serão avaliados inicialmente os aspectos teóricos e as implicações práticas nas implementações de programas com os diversos métodos da resseção iterativos com ângulos de Euler e quatérnios e de métodos diretos com quatérnios e em seguida serão analisados e comparados os resultados dos testes com dados reais obtidos de medições de um tanque cilíndrico vertical com fotogrametria terrestre. 


\section{Rotações com Quatérnios}

Os quatérnios podem ser considerados números hipercomplexos de ordem quatro, que não possuem todas as características de um corpo, uma vez que não admitem comutatividade na multiplicação (Lavieri, 2011). Segundo Wheeler e Ikeuchi (1995), Araújo (2000), Dam et al (1998) e Shih (1990), as rotações com os quatérnios apresentam diversas vantagens: não acontecem situações de instabilidades de soluções; podem ser compostos ou multiplicados de maneira simples para acumular os efeitos das rotações; tem fácil interpretação geométrica; os métodos de interpolações são mais simples e utiliza apenas funções lineares.

As demonstrações das propriedades que se seguem foram desenvolvidas com base em Biasi (2002), Galo e Tozzi (2001), Barreiro (2009), Neves (2008) e Horn (1987).

Os quatérnios podem ser representados através de um vetor com quatro componentes; por uma composição de um escalar e um vetor; ou por um número complexo com três diferentes partes imaginárias, conforme a equação a seguir:

$$
\begin{aligned}
& \dot{q}=\left(q, q_{x}, q_{y}, q_{z}\right) \\
& \dot{q}=q+\vec{q}=(q, \vec{q}) \\
& \dot{q}=q+q_{x} \vec{i}+q_{y} \vec{j}+q_{z} \vec{k}
\end{aligned}
$$

em que: $\dot{q}$ é o quatérnio; $q$ parte real do quatérnio ou primeiro componente do quatérnio; $q_{x}$ segundo componente do quatérnio; $q_{y}$ terceiro componente do quatérnio; $q_{z}$ quarto componente do quatérnio.

Pela relação fundamental em trigonometria há um ângulo em que $q=\cos \theta$ e $|\vec{q}|=\sin \theta$. Então ao considerar $\vec{v}$ um vetor unitário pode-se escrever o quatérnio ainda como:

$$
\dot{q}=\cos \left(\frac{\theta}{2}\right)+\sin \left(\frac{\theta}{2}\right) \vec{v}=\left(\cos \left(\frac{\theta}{2}\right), \sin \left(\frac{\theta}{2}\right) \vec{v}\right)
$$

Então a rotação com quatérnios em relação aos versores $\vec{i}, \vec{j}$ e $\vec{k}$, respectivamente, será expressa por (Pope, 1970):

$$
\begin{aligned}
& R_{Q}(\theta)=\left(\cos \left(\frac{\theta}{2}\right), \sin \left(\frac{\theta}{2}\right) \vec{i}\right) \\
& R_{Q}(\theta)=\left(\cos \left(\frac{\theta}{2}\right), \sin \left(\frac{\theta}{2}\right) \vec{j}\right) \\
& R_{Q}(\theta)=\left(\cos \left(\frac{\theta}{2}\right), \sin \left(\frac{\theta}{2}\right) \vec{k}\right)
\end{aligned}
$$

O ponto $\vec{P}=(x, y, z)$ do espaço tridimensional sobre o qual se efetuará uma rotação será representado pelo quatérnio puro e a rotação será:

$$
R_{q}(\dot{p})=\dot{q} \dot{p} \bar{q}
$$

em que: $\dot{q}$ é o quatérnio; $\dot{p}=(0, \vec{P})$ é o quatérnio puro; $\dot{q}$ é o quatérnio conjugado.

Desenvolvendo a equação 4, por meio das propriedades vetoriais, se tem: 


$$
\begin{aligned}
& R_{q}(\dot{p})=(q, \vec{q})(0, \vec{P})(q,-\vec{q})=(-\vec{q} \vec{P}, q \vec{P}+\vec{q} \times \vec{P})(q,-\vec{q}) \\
& =((-\vec{q} \vec{P}) q-(q \vec{P}+\vec{q} \times \vec{P})(-\vec{q}),(-\vec{q} \vec{P})(-\vec{q})+q(q \vec{P}+\vec{q} \times \vec{P})+(q \vec{P}+\vec{q} \times \vec{P}) \times(-\vec{q})) \\
& =\left((\vec{q} \times \vec{P}) \vec{q} \cdot(\vec{q} \vec{P}) \vec{q}+q^{2} \vec{p}+q(\vec{q} \times \vec{P})+q(\vec{P} \times(-\vec{q}))+(\vec{q} \times \vec{P}) \times(-\vec{q})\right)= \\
& =\left(0,(\vec{q} \vec{P}) \vec{q}+q^{2} \vec{P}+2 q(\vec{q} \times \vec{P})+(\vec{q} \times \vec{P}) \times(-\vec{q})\right)= \\
& =\left(0,2(\vec{q} \vec{P}) \vec{q}+\left(q^{2}-(\vec{q} \vec{q})\right) \vec{P}+2 q(\vec{q} \times \vec{P})\right)
\end{aligned}
$$

Os desenvolvimentos a partir da equação 5 para a representação matricial da rotação com quatérnios são detalhados em Galo e Tozzi (2001). Inicialmente é necessário desconsiderar a parte real da equação 5, por ser nula, o que fica então:

$$
R_{q} \vec{P}=2(\vec{q} \vec{P}) \vec{q}+\left(q^{2}-(\vec{q} \vec{q})\right) \vec{P}+2 q(\vec{q} \times \vec{P})
$$

Desenvolvendo o primeiro termo da equação 6 fica:

$$
2(\vec{q} \vec{p}) \vec{q}=2\left(q_{x} x+q_{y} y+q_{z} z\right)\left(\begin{array}{l}
q_{x} \\
q_{y} \\
q_{z}
\end{array}\right)=2\left(\begin{array}{l}
\left(q_{x} x+q_{y} y+q_{z} z\right) q_{x} \\
\left(q_{x} x+q_{y} y+q_{z} z\right) q_{y} \\
\left(q_{x} x+q_{y} y+q_{z} z\right) q_{z}
\end{array}\right)=2\left(\begin{array}{ccc}
q_{x}{ }^{2} & q_{x} q_{y} & q_{x} q_{z} \\
q_{x} q_{y} & q_{y}{ }^{2} & q_{y} q_{z} \\
q_{x} q_{z} & q_{y} q_{z} & q_{z}{ }^{2}
\end{array}\right)
$$

Para o segundo termo da equação 6:

$$
\left(q^{2}-(\vec{q} \vec{q})\right) \vec{P}=\left(q^{2}-q_{x}{ }^{2}-q_{y}{ }^{2}-q_{z}{ }^{2}\right) \vec{P}=\left(q^{2}-q_{x}{ }^{2}-q_{y}{ }^{2}-q_{z}{ }^{2}\right)\left(\begin{array}{l}
x \\
y \\
z
\end{array}\right)
$$

E para o último termo:

$$
2 q(\vec{q} \times \vec{p})=2 q\left(\left(q_{y} z-q_{z} y\right) \vec{i}+\left(q_{z} x-q_{x} z\right) \vec{j}+\left(q_{x} y-q_{y} x\right) \vec{k}\right)=2 q\left(\begin{array}{ccc}
0 & -q_{z} & q_{y} \\
q_{z} & 0 & -q_{x} \\
-q_{y} & q_{x} & 0
\end{array}\right)\left(\begin{array}{l}
x \\
y \\
z
\end{array}\right)
$$

Desse modo a equação 6 passa a ser escrita como:

$$
R_{z} \vec{p}=2\left(\begin{array}{ccc}
q_{x}^{2} & q_{x} q_{y} & q_{x} q_{z} \\
q_{x} q_{y} & q_{y}^{2} & q_{y} q_{z} \\
q_{x} q_{z} & q_{y} q_{z} & q_{z}^{2}
\end{array}\right)\left(\begin{array}{l}
x \\
y \\
z
\end{array}\right)+\left(q^{2}-q_{z}^{2}-q_{y}^{2}-q_{z}^{2}\right)\left(\begin{array}{c}
x \\
y \\
z
\end{array}\right)+2 q^{2}\left(\begin{array}{ccc}
0 & -q_{z} & q_{y} \\
q_{z} & 0 & -q_{z} \\
-q_{y} & q_{x} & 0
\end{array}\right)\left(\begin{array}{l}
x \\
y \\
z
\end{array}\right)
$$

Na equação 10, após separar $\vec{P}$ formado por $(x, y, z)$, e agrupar os demais termos é obtido $R_{q}$ que é a matriz que representa a rotação quaternária:

$$
R_{q}=\left(\begin{array}{ccc}
q^{2}+q_{x}{ }^{2}-q_{y}{ }^{2}-q_{z}{ }^{2} & 2\left(q_{x} q_{y}-q q_{z}\right) & 2\left(q_{x} q_{z}+q q_{y}\right) \\
2\left(q_{x} q_{y}+q q_{z}\right) & q^{2}-q_{x}{ }^{2}+q_{y}{ }^{2}-q_{z}{ }^{2} & 2\left(q_{y} q_{z}-q q_{x}\right) \\
2\left(q_{x} q_{z}-q q_{y}\right) & 2\left(q_{y} q_{z}+q q_{x}\right) & q^{2}-q_{x}{ }^{2}-q_{y}{ }^{2}+q_{z}{ }^{2}
\end{array}\right)
$$

Mais outros detalhes sobre as propriedades e operações com quatérnios podem ser vistos em Pope (1970) e Horn (1987) além de Galo e Tozzi (2001).

\section{Métodos de Cálculo da Resseção Espacial}

Neste artigo os métodos de resseção espacial em fotogrametria analisados foram classificados da seguinte forma: a) Métodos iterativos, usando a equação da colinearidade com ângulos de Euler e com quatérnios; b) Métodos diretos usando apenas quatérnios. 


\subsection{Métodos iterativos}

\subsubsection{Resseção espacial com a equação da colinearidade e ângulos de Euler}

Neste método a equação da colinearidade é aplicada ao mínimo de três pontos com coordenadas conhecidas e não colineares. No ajustamento é adotado o MMQ, com solução pelo método paramétrico ou combinado e são necessários valores aproximados iniciais para os parâmetros da resseção $\left(\omega, \varphi, \kappa, X_{c}, Y_{c}\right.$ e $\left.Z_{c}\right)$. A Figura 1 mostra a geometria da resseção espacial.

A equação de colinearidade é:

$$
\begin{aligned}
& x=-f \frac{r_{11}\left(X-X_{c}\right)+r_{12}\left(Y-Y_{c}\right)+r_{13}\left(Z-Z_{c}\right)}{r_{\mathrm{s} 1}\left(X-X_{c}\right)+r_{\mathrm{s2}}\left(Y-Y_{c}\right)+r_{\mathrm{sg}}\left(Z-Z_{c}\right)} \\
& y=-f \frac{r_{21}\left(X-\mathrm{X}_{c}\right)+r_{22}\left(\mathrm{Y}-\mathrm{Y}_{c}\right)+r_{23}\left(\mathrm{Z}-\mathrm{Z}_{c}\right)}{r_{\mathrm{s} 1}\left(X-X_{c}\right)+r_{\mathrm{s2}}\left(Y-Y_{c}\right)+r_{\mathrm{ss}}\left(Z-Z_{c}\right)}
\end{aligned}
$$

em que: $\mathrm{x}$ e y são as fotocoordenadas; $\mathrm{f}$ é a distância focal; $\mathrm{X}, \mathrm{Y}$ e $\mathrm{Z}$ são as coordenadas do terreno; $X_{c}, Y_{c}$ e $Z_{c}$, são as coordenadas do centro perspectivo; ${ }^{r_{i j}}$ são os elementos da matriz de rotação, com i e j variando de 1 a 3 .

As matrizes de rotações neste caso são representadas pelos ângulos de Euler. Elas são resultantes do produto de três rotações independentes $\theta_{1}, \theta_{2}, \theta_{3}$ associadas a cada eixo do sistema cartesiano tridimensional respectivamente (x, y e z). Tomando, por exemplo, $\theta_{1}=\omega, \theta_{2}=\varphi$ e $\theta_{3=\kappa}$, as matrizes de rotações independentes $R_{1}(\omega), R_{2}(\varphi)$ e $R_{3}(\kappa)$, no sistema ativo, tem cada um de seus elementos em posições fixas, como estão definidas respectivamente segundo os eixos x, y e $\mathrm{z}$, de acordo os formatos do Quadro1. Então sempre prevalece o formato para $\mathrm{x}, \mathrm{y}, \mathrm{z}$ independentemente de qual seja a sequência das multiplicações.

Quadro 1: Matrizes de rotações independentes para (x, y, z) e $\left(\theta_{1=\omega}, \theta_{2}=\varphi \theta_{3=\kappa}\right)$

\begin{tabular}{|c|cc|c|c|}
\hline \multicolumn{2}{|c|}{$R_{1}(\omega)$ para x } & \multicolumn{2}{|c|}{$R_{2}(\varphi)$ para y } & \multicolumn{2}{|c|}{$R_{3}(\kappa)$ para z } \\
\hline$\left(\begin{array}{ccc}1 & 0 & 0 \\
0 & \cos \omega & -\operatorname{sen} \omega \\
0 & \operatorname{sen} \omega & \cos \omega\end{array}\right)$ & $\left(\begin{array}{ccc}\cos \varphi & 0 & \operatorname{sen} \varphi \\
0 & 1 & 0 \\
-\operatorname{sen} \varphi & 0 & \cos \varphi\end{array}\right)$ & $\left(\begin{array}{ccc}\cos \kappa & -\operatorname{sen} \kappa & 0 \\
\operatorname{sen} \kappa & \cos \kappa & 0 \\
0 & 0 & 1\end{array}\right)$ \\
\hline
\end{tabular}

As matrizes podem ser obtidas por multiplicação pela direita $\left(R_{1} \times R_{2} \times R_{3}\right)$ ou pela esquerda $\left(\mathrm{R}_{3} \times \mathrm{R}_{2} \times \mathrm{R}_{1}\right)$, e ainda segundo o sistema passivo ou ativo. $\mathrm{O}$ produto $\mathrm{R}_{123}=\mathrm{R}_{3} \times \mathrm{R}_{2} \times \mathrm{R}_{1}$ indica que a primeira rotação é a $R_{1}$ (rotação primária), depois é a $R_{2}$ (secundária) e finalmente a $R_{3}$ (terciária.), criando a sequência de rotação 123.

A sequência das rotações pode ter qualquer combinação de multiplicação, mas como o produto de matrizes não é comutativo a sequência das operações afeta a matriz resultante. A seguir é ilustrada como exemplo a matriz de rotação da sequência $(\omega, \varphi, \kappa)$, no sistema ativo.

$$
R_{\text {sex }}=R_{3}(\kappa) \times R_{2}(\varphi) \times R_{1}(\omega)=\left(\begin{array}{ccc}
\cos \kappa \cos \varphi & \cos \kappa \operatorname{sen} \varphi \operatorname{sen} \omega-\operatorname{sen} \kappa \cos \omega & \cos \kappa \operatorname{sen} \varphi \cos \omega+\operatorname{sen} \kappa \operatorname{sen} \omega \\
\operatorname{sen} \kappa \cos \varphi & \operatorname{sen} \kappa \operatorname{sen} \varphi \operatorname{sen} \omega+\cos \kappa \cos \omega & \operatorname{sen} \kappa \operatorname{sen} \varphi \cos \omega-\cos \kappa \operatorname{sen} \omega \\
-\operatorname{sen} \varphi & \cos \varphi \operatorname{sen} \omega & \cos \varphi \cos \omega
\end{array}\right)
$$

Qualquer matriz da forma da equação 13 pode ser comparada diretamente com matriz de rotação com quatérnios (equação 11) e assim podem ser obtidos os ângulos de Euler a partir de quatérnios. Por exemplo, dessa última matriz as rotações $\omega, \varphi$ е қ podem ser obtidos por: 


$$
\begin{aligned}
& \omega=\operatorname{arctg}\left(\frac{r_{32}}{r_{33}}\right) \\
& \varphi=\operatorname{arcsen}\left(-r_{31}\right) \\
& \kappa=\operatorname{arctg}\left(\frac{r_{21}}{r_{13}}\right)
\end{aligned}
$$

em que ${ }^{r_{i j}}$ são os elementos da matriz de rotação com ângulos de Euler.

Vale lembrar que matriz de rotação de quatérnios tem sempre a forma da equação 11 ou de sua transposta, nas quais variam apenas os valores de cada quatérnio, enquanto matrizes como a equação 13 tem inúmeras combinações para cada elemento. Isto implica que a transformação adequada de quatérnio para Euler só pode ser obtida corretamente se a sequência de rotações de Euler for previamente conhecida de algum modo.

Existem os casos de sequência de rotação que podem acarretar o gimbal lock, que é a perda de um grau de liberdade rotacional (Araújo, 2000). Isto pode ser um sério problema em aeronáutica e robótica e que também pode acontecer em fotogrametria, como mostrado em Silva (2014). O problema ocorre quando é escolhida uma sequência de rotações qualquer e há uma situação de alinhamento de dois dos eixos de rotações, de modo que nem todo espaço das rotações possíveis numa próxima sequência poderá ser contemplado (Lavieri, 2011).

Segundo as análises de Silva (2014) é fácil identificar o ângulo possivelmente crítico apenas observando qual a rotação secundária e a composição algébrica dos elementos da matriz de rotação. Ele pode ocorrer em duas situações: a) quando o cosseno do ângulo de Euler da rotação secundária é zero (isto é, quando um dos ângulos de Euler é igual ou próximo de $90^{\circ}$ ou $270^{\circ}$ ), para o caso de rotações realizadas em torno dos três eixos; ou b) em se tratando de rotações realizadas em torno de dois eixos, quando o seno da rotação secundária é zero (ou seja, quando um desses ângulos é igual ou próximo de $0^{\circ}$ ou $180^{\circ}$ ). Com estas situações e valores para os ângulos secundários as rotações desejadas ou previstas podem não ocorrer.

\subsubsection{Resseção espacial com equação da colinearidade e quatérnios}

A resseção espacial também pode ser obtida a partir da equação da colinearidade com substituição das rotações com ângulos de Euler por quatérnios com o seguinte modelo (Jiang et al,2010):

$$
\begin{aligned}
& x=-f \frac{\left(q^{2}+q_{x}^{2}-q_{y}^{2}-q_{z}^{2}\right)\left(x-x_{c}\right)+2\left(q_{x} q_{y}-q_{z}\right)\left(\gamma-\gamma_{c}\right)+2\left(q_{x} q_{z}+q_{y}\right)\left(z-z_{c}\right)}{2\left(q_{x} q_{z}-q_{y}\right)\left(x-x_{c}\right)+2\left(q_{y} q_{z}+q_{x}\right)\left(\gamma-\gamma_{c}\right)+\left(q^{2}-q_{x}{ }^{2}-q_{y}{ }^{2}+q_{z}^{2}\right)\left(z-z_{c}\right)} \\
& y=-f \frac{2\left(q_{x} q_{y}+q_{z}\right)\left(x-x_{c}\right)+\left(q^{2}-q_{x}^{2}+q_{y}^{2}-q_{z}{ }^{2}\right)\left(\gamma-\gamma_{c}\right)+2\left(q_{y} q_{z}-q_{x}\right)\left(z-z_{c}\right)}{2\left(q_{x} q_{z}-q_{y}\right)\left(x-x_{c}\right)+2\left(q_{y} q_{z}+q_{x}\right)\left(\gamma-\gamma_{c}\right)+\left(q^{2}-q_{x}{ }^{2}-q_{y}{ }^{2}+q_{z}{ }^{2}\right)\left(z-z_{c}\right)}
\end{aligned}
$$

A determinação dos parâmetros pode ser feita com o MMQ e são necessários valores iniciais para todos eles. A matriz dos coeficientes é formada com base nas derivadas parciais da equação 15 em relação aos parâmetros incógnitos $\left(X_{c}, Y_{c}, Z_{c}, q, q_{x}, q_{y} e q_{z}\right)$ que podem ser encontradas em Jun et al (2008).

O quatérnio inicial pode ser estimado a partir de ângulos de Euler aproximados ou também um quatérnio adequado à geometria da recessão, como por exemplo, $[1,0,0,0]$. 


\subsection{Métodos diretos}

Existem soluções exatas ("closed-form") para o problema da recessão espacial em topografia que foram adaptadas para a fotogrametria, como Hinsken (1988) e Guan et al (2008), em que se usa basicamente a lei dos cossenos para os triângulos.

Com base nessa lei são encontradas as distâncias do centro perspectivo às coordenadas do terreno e depois os demais parâmetros de posição e orientação são obtidos com uma transformação isogonal 3D. A maioria das soluções exatas usa três pontos no espaço objeto sendo exceção o trabalho de Grafarend e Shan (1997) que usa quatro pontos. Esta solução envolve sempre uma equação de terceiro ou quarto grau.

Outros tipos de soluções exatas usam os quatérnios para obtenção das rotações, como em Horn (1987) e Guan et al (2008). Este último tipo, combinado com o método de Grafarend e Shan (1997), formam a solução exata utilizada neste trabalho detalhada a seguir, que tem a vantagem de encontrar os parâmetros da resseção espacial diretamente, sem a necessidade de iterações e de valores iniciais. A Figura 1 ilustra a geometria da resseção usada por Guan et al (2008) com a localização do centro perspectivo, dos pontos do terreno e as coordenadas da imagem.

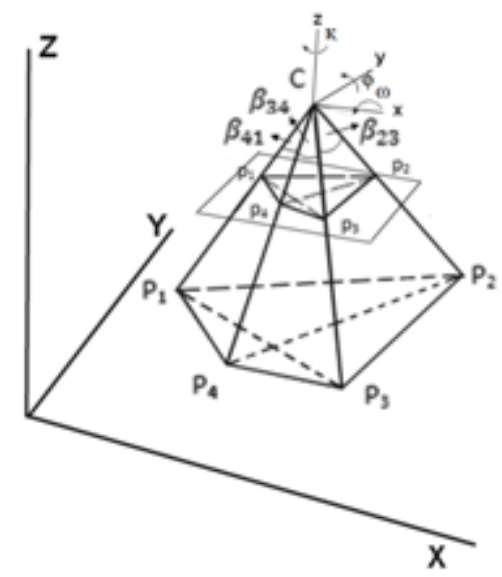

Figura 1: Geometria da resseção espacial. Adaptado de Guan et al (2008).

$\mathrm{Na}$ Figura $1 \mathrm{C}$ representa o centro perspectivo, ${ }_{i}$, com $\mathrm{i}=1, \ldots, 4$, indicam as fotocoordenadas e $P_{i}$ são as coordenadas no espaço objeto; ${ }^{\beta_{i j}}$, são os ângulos espaciais, ou os ângulos entre os sucessivos pontos no espaço objeto.

O cálculo de resseção com este método necessita que sejam previamente conhecidas as distâncias entre o centro perspectivo e cada ponto no terreno. Estas distâncias podem ser obtidas por processo iterativo, com base na lei dos cossenos ou pelo método de Grafarend (Guan et al, 2008). Usando o método de Grafarend (Grafarend e Shan, 1997) o cálculo das distâncias é feito a partir das coordenadas do terreno, das fotocoordenadas respectivas e distância focal.

Com as fotocoordenadas e a distância focal é possível expressar o cosseno dos ângulos espaciais por:

$$
\cos \left(\beta_{i j}\right)=\frac{x_{i} x_{j}+y_{i} y_{j}+f^{2}}{\sqrt{x_{i}^{2}+y_{i}^{2}+f^{2}} \sqrt{x_{j}^{2}+y_{j}^{2}+f^{2}}}
$$


em que: ${ }^{\beta_{i j}}$ são os ângulos espaciais; $\left(x_{i}, y_{i}\right): \mathrm{e}^{\left(x_{j}, y_{j}\right)}$ são as fotocoordenadas; f é a distância focal.

A lei dos cossenos é aplicada em função dos ângulos espaciais e das distâncias requeridas:

$$
\begin{aligned}
& \left\|\overrightarrow{P_{1} P_{2}}\right\|^{2}=\left\|\overrightarrow{C P_{1}}\right\|^{2}+\left\|\overrightarrow{C P_{2}}\right\|^{2}-2\left\|\overrightarrow{C P_{1}}\right\| \mid\left\|\overrightarrow{C P_{2}}\right\| \cos \left(\beta_{12}\right) \\
& \left\|\overrightarrow{P_{2} P_{3}}\right\|^{2}=\left\|\overrightarrow{C P_{2}}\right\|^{2}+\left\|\overrightarrow{C P_{3}}\right\|^{2}-2\left\|\overrightarrow{C P_{2}}\right\|\left\|\overrightarrow{C P_{3}}\right\| \cos \left(\beta_{23}\right) \\
& \left\|\overrightarrow{P_{3} P_{4}}\right\|^{2}=\left\|\overrightarrow{C P_{3}}\right\|^{2}+\left\|\overrightarrow{C P_{4}}\right\|^{2}-2\left\|\overrightarrow{C P_{3}}\right\|\left\|\overrightarrow{C P_{4}}\right\| \cos \left(\beta_{34}\right) \\
& \left\|\overrightarrow{P_{4} P_{1}}\right\|^{2}=\left\|\overrightarrow{C P_{4}}\right\|^{2}+\left\|\overrightarrow{C P_{1}}\right\|^{2}-2\left\|\overrightarrow{C P_{4}}\right\|\left\|\overrightarrow{C P_{1}}\right\| \cos \left(\beta_{41}\right) \\
& \left\|\overrightarrow{P_{1} P_{3}}\right\|^{2}=\left\|\overrightarrow{C P_{1}}\right\|^{2}+\left\|\overrightarrow{C P_{3}}\right\|^{2}-2\left\|\overrightarrow{C P_{1}}\right\|\left\|\overrightarrow{C P_{3}}\right\| \cos \left(\beta_{13}\right) \\
& \left\|\overrightarrow{P_{2} P_{4}}\right\|^{2}=\left\|\overrightarrow{C P_{2}}\right\|^{2}+\left\|\overrightarrow{C P_{4}}\right\|^{2}-2\left\|\overrightarrow{C P_{2}}\right\|\left\|\overrightarrow{C P_{4}}\right\| \cos \left(\beta_{24}\right)
\end{aligned}
$$

Tomando os conjuntos i e j com 4 elementos cada (1 à 4$)$ e fazendo combinações 2 a 2 pode-se

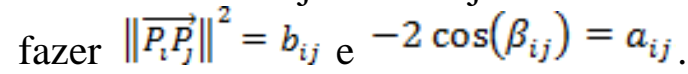

As distâncias finais procuradas correspondem ao módulo dos vetores: $\left\|\overrightarrow{C P_{\imath}}\right\|=x_{i}$, com i variando de 1 à 4.

Com simplificações na equação 17 são obtidas as equações de Grunert (Grafarend e Shan, 1997):

$$
\begin{aligned}
& x_{1}^{2}+x_{2}^{2}+a_{12} x_{1} x_{2}=b_{12} \\
& x_{2}^{2}+x_{3}^{2}+a_{23} x_{2} x_{3}=b_{23} \\
& x_{3}^{2}+x_{4}^{2}+a_{34} x_{3} x_{4}=b_{34} \\
& x_{4}^{2}+x_{1}^{2}+a_{41} x_{4} x_{1}=b_{41} \\
& x_{1}^{2}+x_{3}^{2}+a_{13} x_{1} x_{3}=b_{13} \\
& x_{2}^{2}+x_{4}^{2}+a_{24} x_{2} x_{4}=b_{24}
\end{aligned}
$$

Dessas equações apenas três são independentes. E escolhendo a segunda, terceira e sexta equação de 18 são obtidas novas relações dadas em termos de outras variáveis em coordenadas homogêneas $\mathrm{y}_{3} \mathrm{e} \mathrm{y}_{4}$ :

$$
\begin{aligned}
& y_{3}=\frac{x_{8}}{x_{2}} \\
& y_{4}=\frac{x_{4}}{x_{2}}
\end{aligned}
$$

Isolando-se $x_{2}^{2}$ na segunda, terceira e sexta equação de 18 e usando a equação 19 é obtida a equação:

$$
\begin{aligned}
& x_{2}^{2}=\frac{b_{23}}{1+y_{3}^{2}+a_{28} y_{3}} \\
& x_{2}^{2}=\frac{b_{94}}{y_{3}^{2}+y_{4}^{2}+a_{34} y_{8} y_{4}} \\
& x_{2}^{2}=\frac{b_{24}}{1+y_{4}^{2}+a_{24} y_{4}}
\end{aligned}
$$


Dividindo as duas últimas equações de 20 pela primeira resulta:

$$
\begin{aligned}
& b_{34}\left(1+y_{3}^{2}+a_{23} y_{3}\right)=b_{23}\left(y_{3}^{2}+y_{4}^{2}+a_{34} y_{3} y_{4}\right. \\
& b_{24}\left(1+y_{3}^{2}+a_{23} y_{3}\right)=b_{23}\left(1+y_{4}^{2}+a_{24} y_{4}\right)
\end{aligned}
$$

Resolvendo o sistema formado pela equação 21 , pelo método da adição, é obtido o valor para y4:

$$
y_{4}=\frac{\left(b_{34}-b_{23}-b_{24}\right) y_{3}^{2}+a_{23}\left(b_{34}-b_{24}\right) y_{3}+\left(b_{23}+b_{34}-b_{24}\right)}{a_{34} b_{23} y_{3}-a_{24} b_{23}}
$$

Substituindo agora $\mathrm{y}_{4}$ na equação segunda equação de 21 chega-se a uma equação do quarto grau, em função de $\mathrm{y}_{3}$ :

$$
C_{4} y_{3}^{4}+C_{3} y_{3}^{3}+C_{2} y_{3}^{2}+C_{1} y_{3}+C_{0}=0
$$

em que:

$$
\begin{gathered}
c_{4}=b_{23}\left(b_{34}-b_{23}-b_{24}\right)^{2}-a_{34}^{2} b_{23}^{2} b_{24} \\
C_{3}=\left(b_{34}-b_{23}-b_{24}\right) \times\left[a_{24} a_{34} b_{23}^{2}+2 a_{23} b_{23}\left(b_{34}-b_{24}\right)\right]+a_{34} b_{23}^{2} b_{24}\left(2 a_{24}-a_{23} a_{34}\right) \\
C_{2}=b_{23}\left[a_{23}^{2}\left(b_{34}-b_{24}\right)^{2}+2\left(b_{34}-b_{23}-b_{24}\right)\left(b_{23}+b_{34}-b_{24}\right]+a_{23} a_{24} a_{34} b_{23}^{2}\left(b_{24}+b_{34}\right)\right. \\
+a_{34}{ }^{2} b_{23}^{2}\left(b_{23}-b_{24}\right)+a_{24}{ }^{2} b_{23}^{2}\left(b_{23}-b_{24}\right) \\
c_{1}=2 a_{23} b_{23}\left(b_{34}-b_{24}\right)\left(b_{23}+b_{34}-b_{24}\right)+a_{24} a_{34} b_{23}^{2}\left(b_{34}+b_{24}-b_{23}\right)-a_{23} a_{24}{ }^{2} b_{23}^{2} b_{34} \\
C_{0}=b_{23}\left(b_{23}+b_{34}-b_{24}\right)^{2}-a_{24}{ }^{2} b_{23}^{2} b_{34}
\end{gathered}
$$

Uma vez encontrado o valor de $\mathrm{y}_{3}$ pode ser obtido $\mathrm{x}_{2}$ usando a primeira equação de 20 . Inserindo $\mathrm{y}_{3} \mathrm{e} \mathrm{x}_{2}$ na primeira equação de 19 é obtido $\mathrm{x}_{3}$. Em seguida é obtido y4 com a equação 22 . Com y4 e $\mathrm{x}_{2}$ é obtido $\mathrm{x}_{4}$, pela segunda equação de 19 . Finalmente pode ser obtido $\mathrm{x}_{1}$, com a primeira equação de 18 .

As raízes de 23 são quatro, mas deve ser escolhida como solução um número positivo e real, uma vez que se busca o valor de uma distância. Além disso, foi percebido durante os experimentos, que a grandeza da raiz também influi, ou seja, as raízes devem ser menores que 1,5. Portanto as raízes devem ser testadas por algum procedimento auxiliar que permita definir a raiz "correta".

Obtidas as distâncias $\left(\mathrm{x}_{1}, \mathrm{x}_{2}, \mathrm{x}_{3}, \mathrm{x}_{4}\right)$ os procedimentos finais são os apresentados em Guan et al (2008):

1. Cálculo do fator escala pela relação entre a distância SI (centro perspectivo até as coordenadas do terreno) e a distância em fotocoordenadas, expresso por:

$$
\lambda_{i}=\frac{S t}{\sqrt{x_{i}^{2}+y_{i}^{2}+f^{2}}}
$$

2. Translação das coordenadas para o centro de massa com as equações:

$$
\begin{aligned}
& \overline{p_{i}}=p_{i}-p_{g} \\
& \overline{s_{i}}=s_{i}-s_{g}
\end{aligned}
$$

em que: $p_{i} s_{i}$ são as coordenadas do terreno e fotocoordenadas, respectivamente, com $\mathrm{i}=1, \ldots, \mathrm{n}$; sendo n o total de pontos; $s_{i}, s_{g}$ e $p_{g}$ são dados por: 


$$
\begin{aligned}
& s_{i}=\left(\begin{array}{l}
\lambda_{i} x_{i} \\
\lambda_{i} y_{i} \\
\lambda_{i} f
\end{array}\right) \\
& s_{g}=\frac{1}{n} \sum_{i} s_{i} \\
& p_{g}=\frac{1}{n} \sum_{i} p_{i}
\end{aligned}
$$

O modelo matemático que gera os resíduos é dado por:

$$
V_{i}=R_{q}{ }^{t} p_{i}-s_{i}-T
$$

em que T é obtido por:

$$
T=R_{q}{ }^{t}\left(\begin{array}{l}
X_{c} \\
Y_{c} \\
Z_{c}
\end{array}\right)
$$

4. Formação da matriz $\mathrm{N}$ e cálculo do autovalor:

A matriz $\mathrm{N}$ é o produto de duas matrizes que representam a rotação para um vetor no espaço tridimensional e é apresentada como:

$$
N=\sum_{i}\left(\begin{array}{ccccc}
0 & -x_{p_{i}} & -y_{p_{i}} & -z_{p_{i}} \\
x_{p_{i}} & 0 & -z_{p_{i}} & y_{p_{i}} \\
y_{p_{i}} & z_{p_{i}} & 0 & -x_{p_{i}} \\
z_{p_{i}} & -y_{p_{i}} & x_{p_{i}} & 0
\end{array}\right)^{2}\left(\begin{array}{cccc}
0 & -x_{s_{i}} & -y_{s_{i}} & -z_{s_{i}} \\
x_{s_{i}} & 0 & z_{s_{i}} & -y_{s_{i}} \\
y_{s_{i}} & -z_{s_{i}} & 0 & -x_{s_{i}} \\
z_{s_{i}} & y_{s_{i}} & -x_{s_{i}} & 0
\end{array}\right)
$$

Os elementos que compõem estas matrizes são os quatérnios $P_{i}=\left(0, \bar{p}_{\imath}\right)$ e $S_{i}=\left(0, \bar{s}_{\imath}\right)$.

5. Cálculos complementares:

- Seleção do maior autovalor e cálculo do autovetor correspondente que será o quatérnio.

- Construção da matriz de rotação que deve observar uma sequência de rotação préconhecida.

- Obtenção dos elementos angulares $\omega, \varphi, \kappa$, observando a sequência de rotação equivalente com os quatérnios calculados.

- Cálculo da translação usando a equação 31.

- Cálculo dos demais elementos e das coordenadas do centro perspectivo, que são dadas pelo produto da matriz de rotação com o vetor da translação pela equação:

$$
\left(\begin{array}{c}
X_{c} \\
Y_{c} \\
Z_{c}
\end{array}\right)=T \times R_{q}
$$

\section{Experimentos}

Para os experimentos de resseção espacial foram usados o método tradicional com a equação da colinearidade e dois outros com quatérnios, todos implementados em Matlab®. O método da equação da colinearidade com os ângulos de Euler (COL) foi realizado com o ajustamento pelo método paramétrico, com iterações. 
Os dois métodos com uso de quatérnios são:

- Resseção espacial direta (DIR), que foi implementado seguindo o algoritmo de Guan et al (2008), desenvolvido para usar quatro pontos. Para empregar este método primeiro é necessário calcular as distâncias entre o centro perspectivo e os pontos no espaço objeto, para o qual foi adotado o método de Grafarend e Shan (1997).

- Resseção espacial com iteração (CIT) é uma adaptação da resseção espacial com a equação da colinearidade, com troca da matriz de rotação com ângulos de Euler pelos quatérnios. Neste caso o método tem a desvantagem de necessitar de valores iniciais e tem a vantagem de poder usar mais de quatro pontos.

Neste artigo serão usados apenas os dados de Silva (1995), tanto os dados de entrada como os resultados da fototriangulação, de um levantamento de um tanque cilíndrico, composto por 11 fotografias tomadas no sistema de referência indicado na Figura 2, com X e Z na horizontal e Y na vertical.

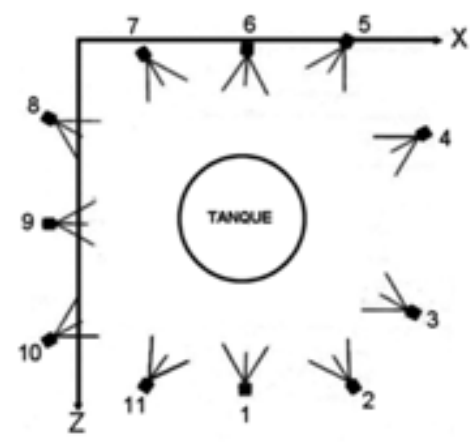

Figura 2:1 Posições das estações de exposição em volta do tanque. Adaptado de Silva (1995).

Os experimentos 1 e 2 são de casos de posições com ambiguidades e em posições críticas. Outros casos e exemplos podem ser encontrados em Silva (2014).

\subsection{Experimento 1}

No experimento 1 é testado um caso já previsto de resultado ambíguo, conhecido a partir da matriz de rotação, que ocorre com a posição da fotografia 5 da Figura 2. A fotografia original tinha vários pontos dos quais foram escolhidos as quatro fotocoordenadas mais bem distribuídas pelos quatro cantos da imagem e apresentadas na Tabela 1.

Tabela 1: Dados de entrada do experimento 1.

\begin{tabular}{|c|c|c|c|c|c|}
\hline & \multicolumn{5}{|c|}{ Coordenadas: } \\
\hline \multirow[t]{2}{*}{$P t$} & \multicolumn{2}{|c|}{ Imagem real (mm) } & \multicolumn{3}{|c|}{ Terreno (m) } \\
\hline & $x$ & $y$ & $X$ & $Y$ & $Z$ \\
\hline 1 & 36,290 & 39,519 & 12,018 & 9,282 & 9,677 \\
\hline 2 & $-34,8$ & 40 & & 9,260 & 13,861 \\
\hline 3 & -50, & $-35,718$ & 21 , & $-0,008$ & 12,631 \\
\hline 4 & 37,500 & $-30,047$ & 12,018 & 9,282 & 9,677 \\
\hline
\end{tabular}

Fonte: Adaptado de Silva (1995).

Com os dados da Tabela 1 foram calculadas as resseções espaciais DIR, CIT e COL, que são apresentadas na Tabela 2. Os resultados mostram que a resseção espacial com a equação da colinearidade não convergiu (NC). A resseção espacial DIR se aproximou do resultado de Silva 
(1995) exceto para o valor de $\varphi$. A CIT apresentou valores próximos para todos os parâmetros exceto para $\varphi$ (segundo ângulo) que resultou em um número complexo.

Tabela 2: Resultado da resseção espacial no experimento 1.

\begin{tabular}{c|c|c|c|c|c|c}
\hline \multicolumn{7}{c}{ Parâmetros aproximados iniciais no método: } \\
\hline & $X_{c_{0}}(m)$ & $Y_{c_{0}}(m)$ & $Z_{c_{0}}(m)$ & $\omega_{0}\left({ }^{\circ}\right)$ & $\varphi_{0}\left({ }^{\circ}\right)$ & $\kappa_{0}\left({ }^{\circ}\right)$ \\
\hline COL & 19,000 & 2,000 & 0,420 & 0,000 & 150,000 & 0,000 \\
\hline DIR & - & - & - & - & - & - \\
\hline CIT & 19,000 & 2,000 & 0,420 & 0,000 & 0,000 & 0,000 \\
\hline \multicolumn{7}{|c|}{ Resultado da resseção espacial com o método: } \\
\hline & $X_{c}(m)$ & $Y_{c}(m)$ & $Z_{c}(m)$ & $\omega\left({ }^{\circ}\right)$ & $\varphi\left({ }^{\circ}\right)$ & $\kappa\left(^{\circ}\right)$ \\
\hline COL & NC & NC & NC & NC & NC & NC \\
\hline DIR & 22,029 & 3,330 & 0,426 & $-3,489$ & 27,074 & 2,360 \\
\hline CIT & 22,020 & 3,340 & 0,420 & $-3,489$ & $-90+24,704 i$ & 2,362 \\
\hline Silva & 22,024 & 3,340 & 0,404 & $-3,420$ & 152,978 & 2,331 \\
(1995) & \multicolumn{7}{|c|}{} \\
\hline COL & - & - & - & - & - & - \\
\hline DIR & $-0,005$ & 0,010 & $-0,022$ & 0,069 & 125,904 & $-0,029$ \\
\hline CIT & 0,004 & 0,000 & $-0,016$ & 0,069 & $-62+24,704 i$ & $-0,031$ \\
\hline
\end{tabular}

No caso da resseção espacial DIR foi observado que arredondando o valor de $\varphi$ e o resultado de Silva (1995) para números inteiros, os ângulos são suplementares e tem a mesma imagem para a função seno. Para solucionar problema como este então deve ser definido o quadrante em que o ângulo se encontra, trocando as funções $\arctan (\mathrm{x}) \operatorname{arcsen}(\mathrm{x})$ da equação 14 pelas funções indicadas por Shih (1990), atan2( $\left.\mathrm{x}_{1}, \mathrm{x}_{2}\right)$ também disponíveis no Matlab®.

A Tabela 3 mostra os novos resultados recalculados, que agora se aproximam do resultado de Silva (1995) para ${ }^{\varphi}$, principalmente a CIT.

Tabela 3: Novos resultados da resseção espacial no experimento 1.

\begin{tabular}{l|c|c|c|c|c|c}
\hline \multicolumn{7}{c}{ Resultado da resseção espacial com o método: } \\
\hline & $X_{c}(m)$ & $Y_{c}(m)$ & $Z_{c}(m)$ & $\omega\left(^{\circ}\right)$ & $\varphi\left(^{\circ}\right)$ & $\kappa\left(^{\circ}\right)$ \\
\hline COL & NC & NC & NC & NC & NC & NC \\
\hline DIR & 22,028 & 3,330 & 0,426 & $-3,489$ & 152,860 & 2,360 \\
\hline CIT & 22,020 & 3,340 & 0,420 & $-3,489$ & 152,968 & 2,362 \\
\hline $\begin{array}{l}\text { Silva } \\
(1995)\end{array}$ & 22,024 & 3,340 & 0,404 & $-3,420$ & 152,978 & 2,331 \\
\hline \multicolumn{7}{|c}{ Diferenças Silva (1995) demais métodos: } \\
\hline COL & - & - & - & - & - & - \\
\hline DIR & 0,005 & $-0,01$ & 0,022 & $-0,069$ & $-0,118$ & 0,029 \\
\hline CIT & $-0,004$ & 0,000 & 0,016 & $-0,069$ & $-0,010$ & 0,031 \\
\hline
\end{tabular}

\subsection{Experimento 2}

O objetivo do experimento 2 é avaliar os métodos implementados, principalmente a resseção espacial sem iteração, em fotografias cujas posições são críticas, isto é, quando ocorrem gimbal lock, como é o caso da fotografia de posição 9 da Figura 2. A posição dessa fotografia é crítica porque ela está com rotação $R_{\kappa \varphi \omega}$, no sistema passivo, com o ângulo da rotação secundário $\varphi$ próximo de $270^{\circ}$. Na Tabela 4 são mostrados os dados de entrada deste experimento. Os resultados são mostrados na Tabela 5 . 
Tabela 4: Dados de entrada do experimento 2.

\begin{tabular}{c|c|c|c|c|c}
\hline & \multicolumn{5}{|c}{ Coordenadas: } \\
\hline \multirow{2}{*}{$\boldsymbol{P t}$} & Imagem real (mm) & \multicolumn{3}{|c}{ Espaco objeto (m) } \\
\cline { 2 - 6 } & $\boldsymbol{x}$ & $\boldsymbol{y}$ & $\boldsymbol{X}$ & $\boldsymbol{Y}$ & $\boldsymbol{Z}$ \\
\hline $\mathbf{1}$ & 40,064 & 49,968 & 9,775 & 9,247 & 20,604 \\
\hline $\mathbf{2}$ & $-38,565$ & 53,280 & 9,228 & 9,243 & 11,534 \\
\hline $\mathbf{3}$ & $-42,934$ & $-34,928$ & 7,515 & $-0,103$ & 11,946 \\
\hline $\mathbf{4}$ & 33,021 & $-42,275$ & 5,944 & $-0,027$ & 18,660 \\
\hline
\end{tabular}

Tabela 5: Resultado da resseção espacial no experimento 2.

\begin{tabular}{|c|c|c|c|c|c|c|}
\hline \multicolumn{7}{|c|}{ Parâmetros aproximados iniciais no método: } \\
\hline & $X_{c_{0}}(\mathrm{~m})$ & $\hat{Y}_{c}(\mathrm{~m})$ & $Z_{c_{2}}(\mathrm{~m})$ & $\omega_{0}\left({ }^{\circ}\right)$ & $\varphi_{0}\left({ }^{\circ}\right)$ & $\kappa_{0}(\mathrm{C})$ \\
\hline $\mathrm{COL}$ & $-2,400$ & 2,000 & 17,000 & 25,000 & 265,000 & 28,00 \\
\hline DIR & 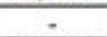 & - & 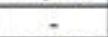 & 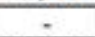 & 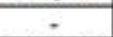 & - \\
\hline CIT & $-2,400$ & 2,000 & 17,000 & 0,500 & 100,760 & 0,570 \\
\hline \multicolumn{7}{|c|}{ Resultado da resseção espacial com o método: } \\
\hline & $X_{c}(\mathrm{~m})$ & $Y_{s}(m)$ & $Z_{c}(m)$ & $\omega(\circ)$ & $\varphi(\odot)$ & $x\left(0^{\circ}\right)$ \\
\hline $\mathrm{COL}$ & $\mathrm{NC}$ & NC & $\mathrm{NC}$ & NC & NC & $\mathrm{NC}$ \\
\hline DIR & $-1,984$ & 3,250 & 16,051 & 29,520 & $-89,560$ & 30,364 \\
\hline CIT & $-1,983$ & 3,245 & 16,054 & 26,238 & $-90,475$ & 27,108 \\
\hline Iva (1995) & $-1,997$ & 3,251 & 16,053 & 26,682 & 270,439 & 27,524 \\
\hline \multicolumn{7}{|c|}{ Diferenças Silva (1995) demais métodos: } \\
\hline $\mathrm{COL}$ & 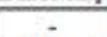 & - & 2 & $=$ & - & $\theta$ \\
\hline DIR & 0,013 & $.0,001$ & $-0,002$ & 2838 & 0,001 & 2,840 \\
\hline
\end{tabular}

\section{Discussão dos Resultados}

O experimento 1 mostrou que pode haver ambiguidades nos resultados da resseção espacial, mesmo usando quatérnios, seguido da conversão para ângulos de Euler. Para resolver este problema foi necessário introduzir no programa as funções trigonométricas de Shih (1990), que permite extrair o valor do ângulo da rotação secundária no quadrante correto. Com isso as diferenças com relação aos demais parâmetros ficam bem pequenas, ao serem comparados com o resultado da fototriangulação.

Os resultados do experimento 2, relacionados na Tabela 5, mostram que a resseção COL não converge, fato que já era esperado por envolver uma posição classificada como crítica. Já a resseção espacial DIR apresentou valores bem semelhantes para as coordenadas do centro perspectivo, enquanto que para os ângulos existem diferenças em torno de $3^{\circ} \mathrm{em}{ }^{\omega} \mathrm{e} \mathrm{k}$. Essas diferenças podem ser por conta dos erros aleatórios de uso apenas de quatro coordenadas, enquanto que na fototriangulação foram utilizadas muito mais pontos. $O$ valor obtido de $\varphi=$ $270,439^{\circ}$, é equivalente a $-89,561^{\circ}$, por isso a diferença de $0,001^{\circ}$ do resultado de Silva (1995).A Tabela 5 também mostra que a resseção CIT ficou bem semelhante ao resultado de Silva (1995) e bem melhor que os resultados da resseção DIR.

Resseções espaciais de outras fotografias críticas foram testadas com dados simulados de Silva (2014), com os três métodos e também só foram obtidos resultados corretos com os quatérnios, o que confirma as recomendações de Araújo (2000) e Shih (1990).

Nos dois experimentos os parâmetros iniciais estão nas primeiras linhas das Tabelas 2 e 5. Para a resseção com os métodos com colinearidade, Euler e Quatérnios, os valores são todos iguais para a posição, e mudam para os ângulos, notando que para o método com quatérnio (CIT) os ângulos podem ser muito menos aproximados. Note-se que mesmo colocando valores iniciais bem aproximados nos experimentos 1 e 2 para a colinearidade com Euler (COL) não houve convergência. $O$ valor de $\varphi$ inicial igual a 150,0 (quando da fototriangulação o valor é 152,978) 
no experimento 1, e igual a 265,0 no experimento 2 (na fototriangulação igual a 270,439) apenas convergiu com CIT. Para o método direto (DIR) não há necessidade de valores iniciais, mas são necessárias as distâncias obtidas com o método de Grafarend.

O critério de convergência adotado nos métodos com iterações (COL e CIT) foram a estabilização dos resíduos ( ${ }^{t} \mathrm{PV}$ ) e sempre com os mesmos limiares, sendo que na resseção com quatérnio (CIT) o número de iterações é sempre menor.

\section{Conclusões}

Os resultados finais dos testes com a substituição dos ângulos de Euler por quatérnios na resseção espacial em fotogrametria comprovam que os métodos (DIR e CIT) com quatérnios dão soluções mais estáveis e mais confiáveis. Para o método direto (DIR) as conclusões e recomendações mais importantes são:

- O método auxiliar implementado para fornecer as distâncias entre o centro perspectivo e os pontos no terreno, o de Grafarend, é muito sensível a pequenos erros aleatórios nas coordenadas da imagem e do terreno. Também não é de uso simples e direto, porque usa raízes de um polinômio de quarto grau e necessita de implementação de testes auxiliares para encontrar a solução correta.

- Tem a grande vantagem de não necessitar de valores iniciais para ângulos e centro perspectivo, que podem ser muito difíceis de serem obtidos nos casos de fotografias inclinadas e posições não comuns da fotogrametria terrestre.

Para o método com iterações (CIT) as conclusões e recomendações mais importantes são:

- Pode ser implementado para mais de quatro pontos, o que aumenta a confiabilidade do resultado.

- Necessita de valores iniciais adequados mesmo para os quatérnios. De modo geral os valores iniciais não precisam ser tão bons quanto ao usar os ângulos de Euler na resseção com a equação da colinearidade comum e converge mais rapidamente.

\section{AGRADECIMENTOS}

Os autores agradecem o apoio financeiro da CAPES que permitiu o desenvolvimento deste trabalho.

\section{REFERÊNCIAS BIBLIOGRÁFICAS}

Araújo, Edson L. "Interpolação de Rotações de Objetos Sólidos via Quatérnios". Dissertação de Mestrado, Universidade Federal da Paraíba, 2000.

Barreiro, Rodrigo C. "Quatérnios, um ensaio sobre a regularidade e hiperperiodicidade de funções quaterniônicas, e o Teorema de Cauchy”. Dissertação de Mestrado, Universidade Estadual Paulista, 2009.

Biasi, Sergio. C. "Utilização de Quaternios para a representação de rotações em 3D". Monografia de Graduação, Pontíficia Universidade Católica do Rio de Janeiro, 2002. Acessado em: 23 de Julho, 2011. http://webserver2.tecgraf.puc-rio.br/ mgattass/Quaternios.pdf. 
Dam, Erik B., Koch, Martin e Lillholm, Martin. Quaternions, interpolation and animation. Cambridge: Massachusetts Institute of Technology, 1998.

Galo, Maurício e Tozzi, Clésio L. "A representação de matrizes de rotação e o uso de quatérnios em ciências geodésicas”. In Série em Ciências Geodésicas. Editor Mitishita, Edson A.Vol.1 p. 214-231. Curitiba: UFPR, 2001.

Grafarend, E. e Shan. J. "Closed-form solution of P4P or three-dimensional resection problem in terms of Mobius barycentric coordinates”. Journal of Geodesy. 71 (1997): 217-231.

Guan, Yunlan, Cheng, Xiaojun, Zhan, Xinwu e Zhou, Shijian. "Closed-form solution of space resection using unit quaternion". Artigo apresentado no XXI ISPRS Congress, Beijing, China, 311 de julho, 2008. Acessado em: 05 de Dezembro de 2012. http://www.isprs.org/proceedings/XXXVII/congress/3b_pdf/09.pdf

Hinsken, Ludger. "A singularity free algorithm for spatial orientation of bundles". Artigo apresentado no XVI ISPRS Congress, Kyoto, Japan. 1-10 de julho, 1988. Acessado em: 05 de Dezembro de 2012. http://www.isprs.org/proceedings/xxvii/congress/part5/262_XXVIIpart5.pdf.

Horn, Berthold. "Closed-form solution of absolute orientation using unit quaternions". Journal of the optical Society of America. 4 (1987): 629-642. Acessado em: 25/Out/2012. http://people.csail.mit.edu/bkph/papers/Absolute_Orientation.pdf

Jun, Liu, Donghong, Wang e Yongsheng, Zhang. "Triangulation of airborne three-line images using quaternion". Artigo apresentado no XXI ISPRS Congress, Beijing, China, 3-11 de julho, 2008. Acessado em: 05 de Dezembro de 2012. http://www.isprs.org/proceedings/XXXVII/congress/1_pdf/97.pdf.

Lavieri, Rodrigo S. "Métodos de navegação inercial aplicados a lançamentos submarinos". Dissertação de Mestrado, Universidade de São Paulo, 2011.

Lugnani, João B. Introdução à fototriangulação. Curitiba: Imprensa Universitária, 1987.

Neves, Robson C. "Os quatérnios de Hamilton e o Espaço". Dissertação de Mestrado. Universidade Federal do Rio de Janeiro, 2008.

Pope, Allen J. An advantageous, alternative parameterization of rotations for analytical photogrammetry. ESSA Technical Report C\&GS 39. Rockville, Maryland: U.S. Department of Commerce, 1970.

Shih, Tian-Yuan. "The Duality and Critical Condition in the Formulation and Decomposition of a Rotation Matrix". Photogrammetric Engineering \& Remote Sensin. Vol. 56, n. 8, p. 11731179, Aug. 1990. Maryland: American Society for Photogrammetry and Remote Sensing, 1990.

Silva, Amanda M. "A Representação das Matrizes de Rotações com o Uso dos Quatérnios: Aplicações à Fotogrametria". Dissertação de Mestrado, Universidade Federal de Pernambuco, 2014.

Silva, Daniel C. "Considerações Práticas em Fotogrametria a Curta Distância Aplicada ao Levantamento de um Tanque e a Questão da Precisão e Exatidão". Dissertação de Mestrado, Universidade Federal do Paraná, 1995.

Wheeler, Mark D e Ikeuchi, Katsushi. Iterative Estimation of Rotation and Translation using the Quaternion CMU-CS-95-215. Pittsburgh: Carnegie Mellon University, 1995.

Recebido em Junho de 2014. Aceito em Agosto de 2015. 